\title{
A Cadaveric Investigation to Advance the Understanding of Deltoid Vein
}

\author{
Sunita Kalra, ${ }^{1}$ Gitanjali Khorwal, ${ }^{2}$ Shweta Jha, ${ }^{3}$ Sonu ${ }^{4}$ \\ 'Department of Anatomy,University College of Medical Sciences, Delhi-110095 \\ 2Department of Anatomy, AllMS, Rishikes \\ ${ }^{3}$ Department of Anatomy, Heritage Institute of Medical Science, Varanasi-221311 \\ ${ }^{4}$ Department of Anatomy, University College of Medical Sciences, Delhi-110095
}

Disclose and conflicts of interest: none to be declared by all authors

\section{ABSTRACT}

Introduction: descriptions of the tributaries of axillary vein including thoracoacromial and cephalic veins are significant clinically however usually are terse and deficient in detail in standard textbooks of anatomy. The knowledge about anatomy of these venous structures is deficient as well as complicated. The chief objective of the present study was to establish the presence of deltoid vein in the deltopectoral groove, its relations in the immediate vicinity and establish any variations which can evaluate its feasibility for catheter implantation for central venous access.

Materials and methods: the study was conducted over 12 (24 sides) formalin fixed cadavers perfused with formalin based fixative. After removal of skin flap from the deltopectoral region, deep fascia in the deltopectoral groove was divided to expose the cephalic vein and other structures in deltopectoral triangle.

Results: structures including cephalic vein ,thoracoacromial artery and vein were identified in the immediate vicinity of deltopectoral triangle. Deltoid branch of thoracoacromial artery was identified close to deltoid vein. The topography and relations of deltoid vein were studied. It was traced till its drainage into recipient vessel.

Conclusion: in our study we have studied the relations of cephalic and deltoid veins in the deltopectoral groove which can be useful for successful catheter implantation and prevent damage to these important venous structures with scarce data on their topography.

Key words: Cephalic vein; Deltoid vein; Thoracoacromial vessels; Delto pectoral triangle.

\section{Introduction}

Descriptions of the tributaries of axillary vein including thoracoacromial and cephalic veins are significant clinically however usually are terse and. deficient in detail in standard textbooks of anatomy ${ }^{1-3}$ The knowledge about anatomy of these venous structures is deficient, complicated and is subjected to wide variety of variations although being of enormous importance for various pathological conditions and surgical interventions. Frequently central venous access is achieved through the subclavian, femoral, brachiocephalic or cephalic veins. ${ }^{4}$ Central venous catheters in the upper limb and head and neck, are inserted conventionally, "blindly" through diverse anatomic landmarks such as the clavicle, sternal notch, sternocleidomastoid muscle and the carotid artery. Countless life saving interventions requires surgical exposure to cannulate these in critical care units. Familiarity about encountering various variations as provided in our study with reference to these venous structures is going facilitate immense assistance.

The study involved twenty four upper limbs of formalin fixed cadavers which were being used for undergraduate teaching in a medical institute. The axillary vein is the continuation of the basilic vein. It begins at the lower border of teres major and ascends to the outer border of the first rib, where it becomes the subclavian vein. It is joined by the brachial vein near subscapularis, and by the cephalic vein near its costal end; other tributaries follow similar to axillary arterial branches, namely superior thoracic, thoracoacromial, lateral thoracic, subscapular, anterior and posterior circumflex humeral. The axillary vein lies medial to the axillary artery, which it to some extent overlaps. The second part of axillary artey gives a branch named as thoraco-acromial artery. It is partly overlapped by pectoralis minor muscle near its medial border and after piercing clavipectoral fascia, thoraco acromial artery gives four terminal branches namely deltoid , pectoral, acromial and clavicular.

The thoraco-acromial vein is comparable to thoracoacromial artery and its tributaries follow the similar course. Deltoid vein is one of the four named tributaries of thoracoacromial vein along with acromial, pectoral and clavicular veins. Thoracoacromial vein ultimately drains into axillary vein. ${ }^{3}$

The aim of our study was to access data regarding the topography and the associations of the deltoid 
vein, as it relates to the deltopectoral triangle. A cadaveric investigation was performed to advance the anatomical understanding of this relationship. The chief purpose of present study was to establish the presence of deltoid vein in the deltopectoral groove, its relations in the immediate vicinity and establish any variations which can evaluate its feasibility for catheter implantation for central venous access.

\section{Materials and Methods}

The study was conducted over 12 (24 sides) formalin fixed cadavers perfused with formalin based fixative .The cadavers were of either sex (10 male, 2 female) which were being used for routine dissection purposes for teaching undergraduate medical students at a Medical Institute. Dissection was carried out according to the dissection steps given in Cunningham's manual of practical anatomy and their pectoral regions were carefully dissected. ${ }^{5}$ After removal of skin flap from the deltopectoral region, deep fascia in the deltopectoral groove was divided to expose the cephalic vein.

Clavicular head of pectoralis major muscle was cut and reflected toward its insertion. Structures including cephalic vein, thoracoacromial artery and vein were identified in the immediate vicinity. Deltoid branch of thoracoacromial artery was identified close to deltoid vein. The topography and relations of deltoid vein were studied. It was traced till its drainage into recipient vessel. Cephalic vein was also traced till its drainage into axillary vein. All the findings were recorded and analyzed.

\section{Results}

The total of 24 upper limbs were studied and it was found that in all i.e. in $100 \%$ limbs both the deltoid vein and cephalic vein were identified effortlessly in the deltopectoral region (Figure. 1-4). Cephalic vein was located to be lateral most structure and deltoid vein was found to be positioned medial to it but for one case in which deltoid vein was changing position from lateral to medial side of cephalic vein (Figure 3). Although interrelation between cephalic vein and deltoid vein was maintained, relation of other structures in the vicinity was quite variable. Cephalic vein was found to be tremendously thin and fibrosed in two (16.7\%) of the limbs studied (Figure 1). Drainage pattern of even deltoid vein was variable. In approximately $92 \%$ it drained into thoracoacromial vein and subsequently thoracoacromial vein drained into axillary vein (Figure $1,3)$. In one out off a total of twenty four limbs studied, deltoid and cephalic veins both joined via a $3 \mathrm{~cm}$ long venous channel which eventually drained as a single vein into axillary vein (Figure 2). In this case definite thoracoacromial vein was not discovered to form. Even pectoral vein drained directly into axillary vein. In one case $(8.3 \%)$ deltoid vein even joined with pectoral vein and drained directly into axillary vein (Figure 4). In this cephalic vein as well as deltoid vein also demonstrated significant increase in caliber before draining into axillary vein. The location of deltoid vein was not constant in relation to its corresponding arterial counterpart. Deltoid vein was found to be inconsistent in position and was identified either medial or lateral

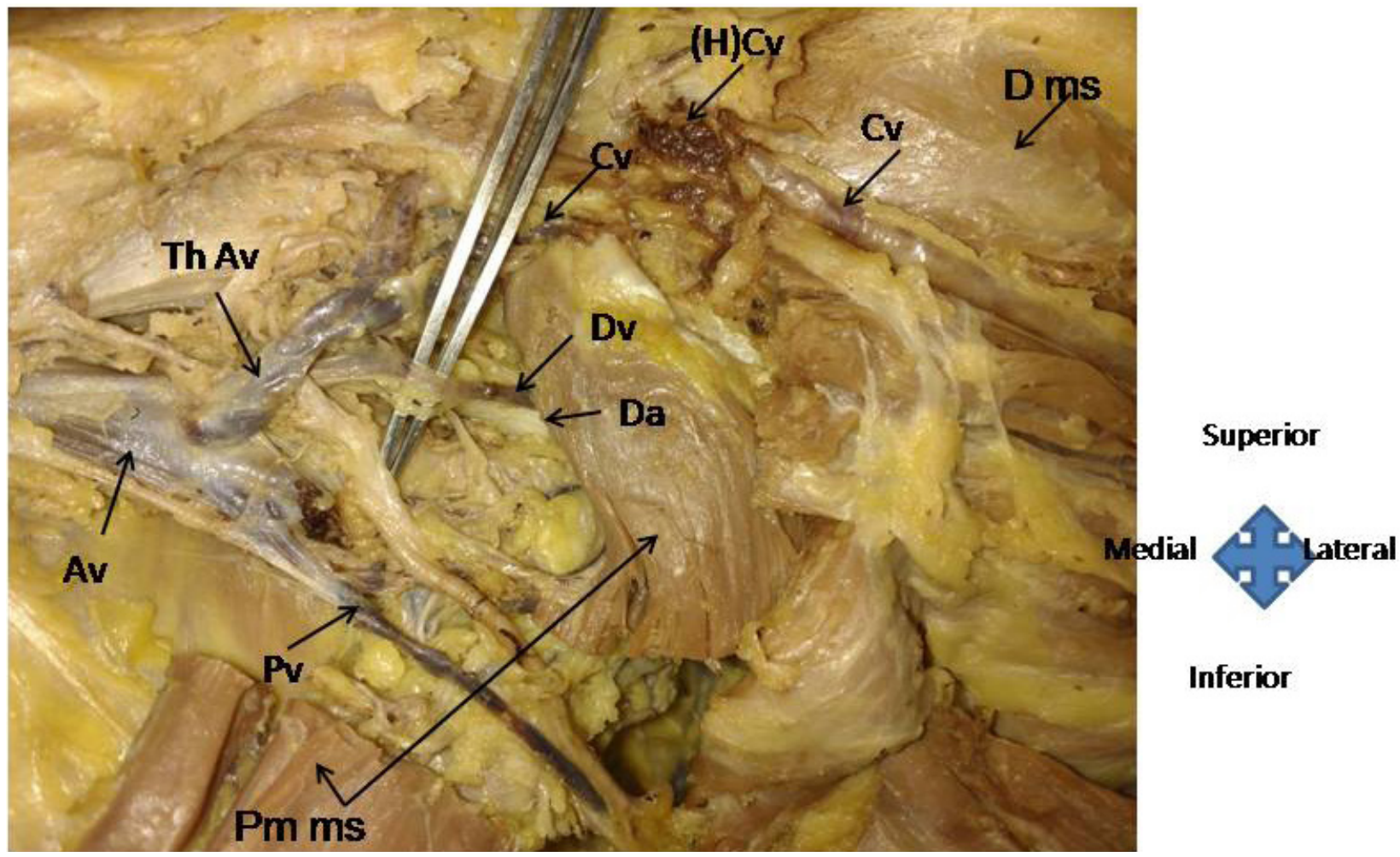

Figure 1. Dissection of left delto -pectoral region showing: Cephalic vein (Cv) thin \& fibrosed. Axillary vein (Av), Thoraco acromial vein (Th Av). Deltoid vein (Dv) and pectoral vein $(\mathrm{Pv})$. 


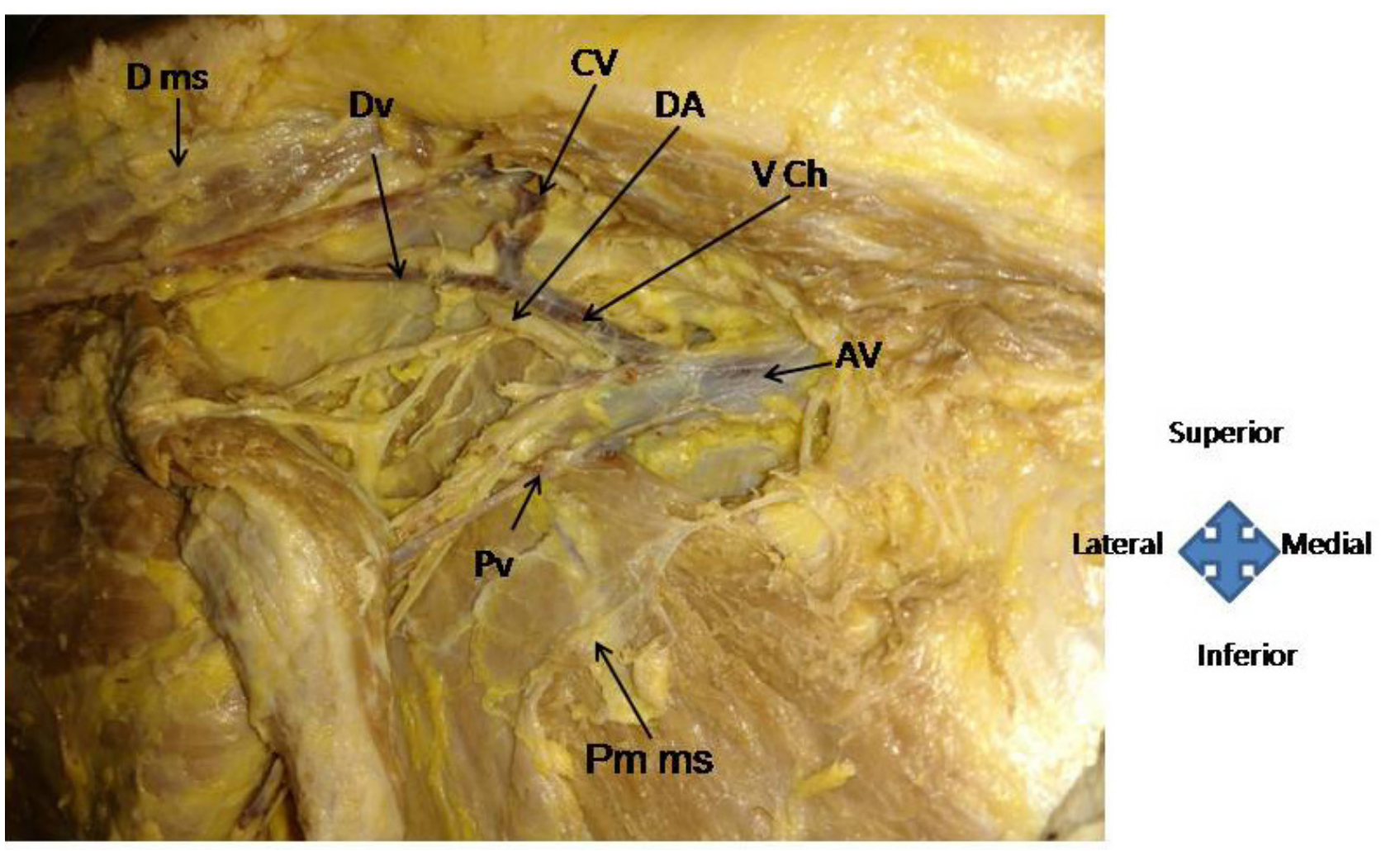

Figure 2. Dissection of right delto - pectoral region showing: Deltoid $v(\mathrm{Dv}) \&$ cephalic $\mathrm{v}(\mathrm{Cv})$ joined by $3 \mathrm{~cm}$ long venous channel $(\mathrm{V} \mathrm{CH})$ which is eventually draining into Axillary vein (Av). Pectoral vein (Pv)also draining directly into axillary vein (Av). Definite Thoraco acromial vein is not formed

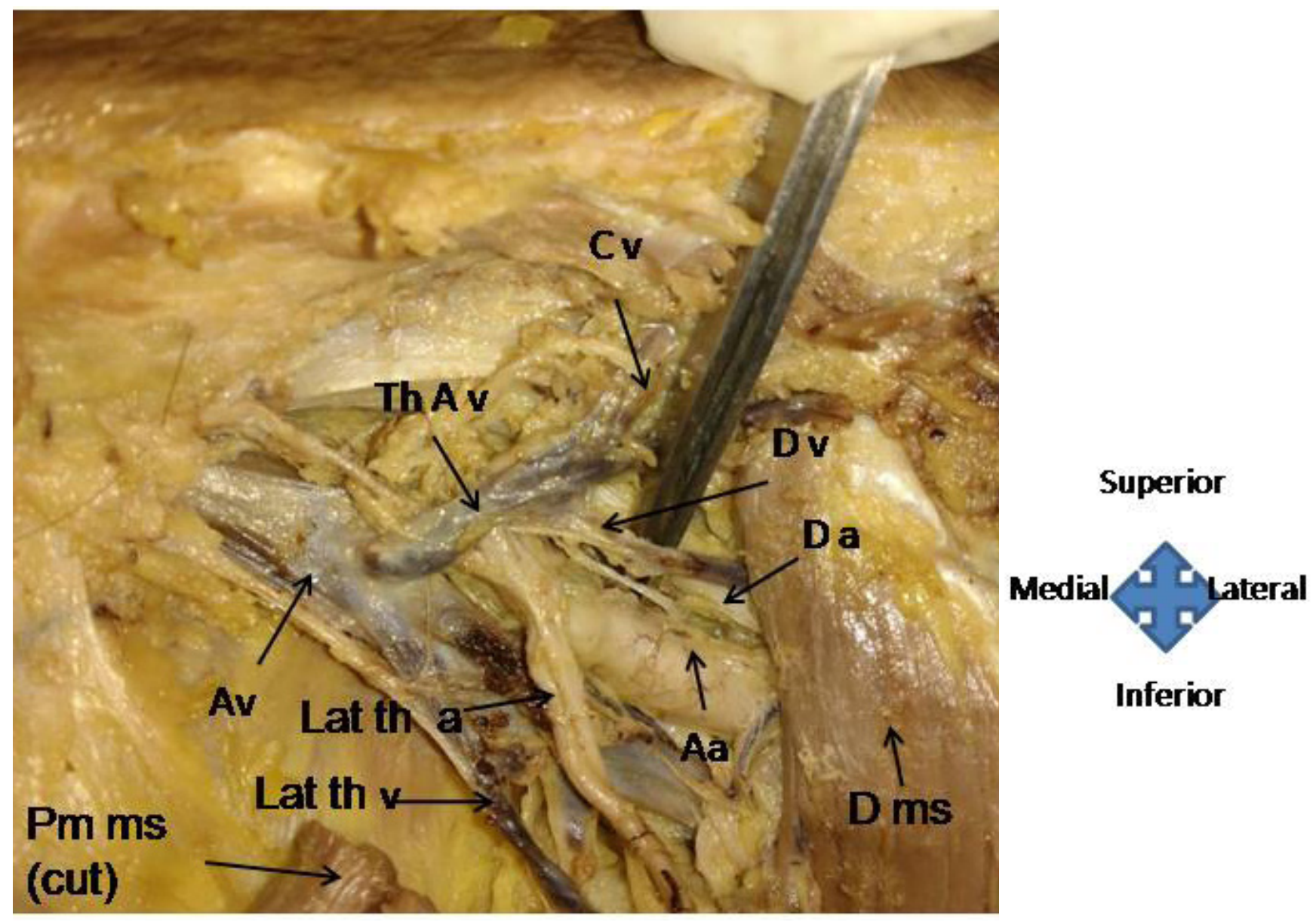

Figure 3. Dissection of left delto -pectoral region showing: Deltoid vein (Dv) is lateral to its counterpart artery draining into thoraco-acromial vein (Th A v). Cephalic vein $(C v)$ is lateral most structure. Axillary artery (Aa) Pectoral vein is seen (P v). 


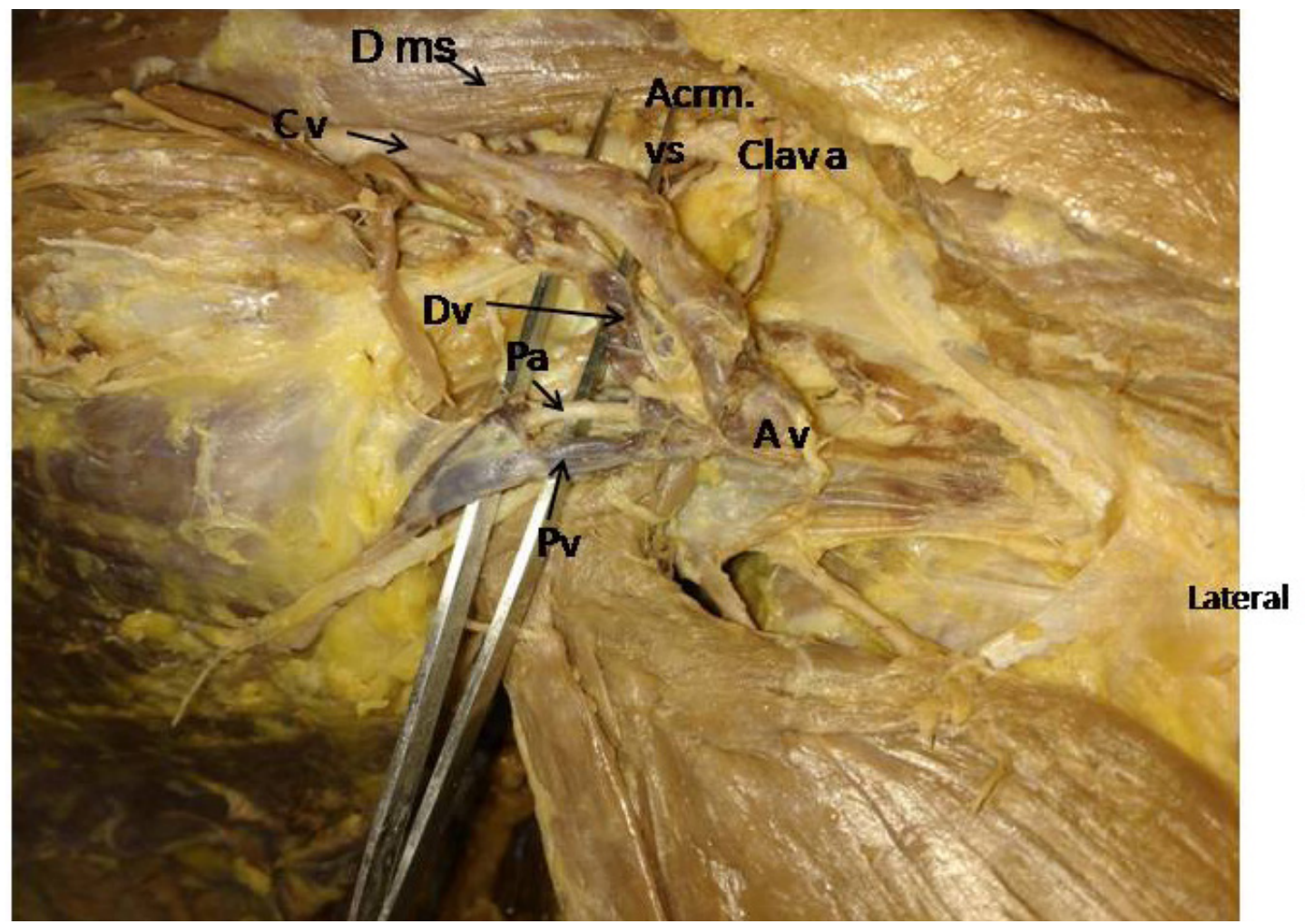

Superior

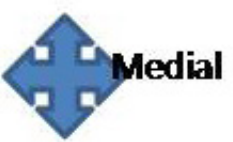

Inferior

Figure 4. Dissection of right delto -pectoral region showing: Cephalic vein \& deltoid vein. increasing caliber draining into Axillary vein (Av) Deltoid artery lateral(D A) Deltoid vein (DV) and pectoral vein ( $P$ v) join \& drain into axillary vein (A v)

to deltoid arterial branch of thoracoacromial artery. In 8.3\%, deltoid vein even crossed superficial to deltoid artery, traversed from its medial to lateral side and subsequently changed its location.

\section{Discussion}

The prospective analysis of the cephalic vein cut down approach in 100 cancer patients for chronic indwelling central venous access was done in a study. The authors suggested that although cephalic vein was the most regular and easiest option because of its relatively consistent location and large caliber. However, it was observed that the cephalic vein could not be identified in almost $18 \%$ of patients and that necessitated a need different entry vessel for implantation of central venous catheter. ${ }^{6}$

The other common substitute entry vessels for catheter implantation include the subclavian and internal jugular veins. It has been suggested that when catheter implantation is attempted through the subclavian vein, there is critical risk of hemopneumothorax and even pinch-off symptoms cannot be completely eliminated even with a skilled surgeon. ${ }^{7-10}$ When the internal jugular vein is used for catheter implantation the risk of inadvertent vessel injury still vestiges even under echo-guidance and may necessitate vessel repair in many cases. ${ }^{11}$ Therefore, the subclavian vein or internal jugular veins are not perfect entry vessels for catheter implantation. Henceforth, a safe alternative entry vessel that can be easily explored is crucial for patients without feasible cephalic vein or for those who need port reimplantation because of disease relapse. Our study suggests that this life threatening damage to internal jugular vein is completely preventable if another safer vessel such as deltoid vein is used for catheter implantation. To authors of present study it appears that the primary reason for deltoid vein not being used commonly for catheter implantation is insufficient availability of anatomical studies about it.

Similar to our proposal, it has been suggested by some researchers that the deltoid branch of the thoracoacromial artery and vein are located in the neighborhood of the cephalic vein in the deltopectoral groove and may be used as recipient vessels of reconstructed muscle. ${ }^{12-14}$ In another exception with changing relations.

The goal of our study was to identify the location and pattern of the deltoid branch of the thoracoacromialvein and evaluate its feasibility for catheter implantation. The previous study ${ }^{16}$ identified the location of the deltoid branch of the thoracoacromial vessel in the deltopectoral groove, with the majority (91\%) of vessel patterns consisting of 1 artery and 1 vein. Due to variations in anatomy, a suitable deltoid branch of the thoracoacromial vein for catheter implantation could not be identified in almost $9 \%$ of patients. In the study, ${ }^{17}$ 9.7\% of patients underwent catheter implantation via the deltoid branch of the thoracoacromial vein due to disease or fibrotic cephalic vein, possibly linked to 
aforementioned anatomic variation. The implantation success rate was $100 \%$ where a feasible deltoid branch of the thoracoacromial vein was identified. The location of the deltoid branch of the thoracoacromial vein has been further clarified. In this study, only $14.2 \%$ of patients had cephalic vein located with the deltoid branches of the thoracoacromial vessel, while the majority $(85.8 \%)$ of patients were found to have the deltoid branch of the thoracoacromial vessel at the medial aspect of the deltopectoral groove, beneath the pectoralis major muscle, which is in conformity of our study.

However, a cadaveric study ${ }^{16}$ demonstrated that $65.2 \%$ of the cephalic vein travels with the deltoid branches of the thoracoacromial trunk. This difference may be caused by the dehydration effect of a preservative such as formaldehyde that is used in the preparation of the cadaver. The high percentage of occurrence and relative constant location revealed that the deltoid branch of the thoracoacromial vein could be considered an alternative candidate for catheter implantation.

In the present study, we have not only demonstrated constantrelation of deltoid tributary of thoracoacromial vein with cephalic vein but have reported various variations namely, fibrosed cephalic vein, absence of thoracoacromial vein and development of a well defined common venous channel between cephalic vein and deltoid vein draining into axillary vein.

The anatomic possibility of variations of thoracoacromial vein and its tributaries especially deltoid vein as provided in the present study can provide essential pre procedural assessment of risk and prevent inevitable failure of implantation of catheters or the inestimable risk associated with complications of it. This study can further ensure that there are minimal or even no inevitabilities whenever central venous catheter implantation is attempted through deltoid vein into thoracoacromial vein in deltopectoral region.

\section{Conclusions}

Knowledge of the topography of cephalic and deltoid veins in the deltopectoral region is important for catheter implantation as any deviation/ injury may not only lead to failed catheter implantation, development of accidental fistulas but excessive bleeding may ensue and cause even delayed healing in case of reconstructive surgeries. Detailed knowledge of the normal and variant anatomy of these veins will be valuable to assess accessibility and achievability for the same. Inconsistency of these veins is also significant for clinicians and radiologist for investigations and diagnosing diverse pathologies in this region. It is anticipated that this study will be useful to surgeons for pre-procedural planning and for clinicians and radiologist to prevent misdiagnosis. In our study we have studied the relations of cephalic and deltoid veins in the deltopectoral groove which can be useful for successful catheter implantation and prevent damage to these important venous structures with scarce data on their topography.

\section{References}

1. Clemente CD .Gray's anatomy. 30th American edition,1985; The Veins, Lea and Febiger, Philadelphia, 820-821.

2. Moore KL, Dalley AF II .Clinically oriented anatomy. 5th ed. Lippincott Williams \& Wilkins, Baltimore,2006; 748-749.

3 Susan Standring. Gray's Anatomy. 41st edition. Elsevier: 2016; 828-830.

4. LeDonne J. Percutaneous cephalic vein cannulation (in the deltopectoral groove), with ultrasound guidance. J Am Coll Surg,2005; 810-811.

5. Romans GJ .Cunningham's manual of practical anatomy. 15th edition (volume 1). Oxford university press, 1986; 22-28.

6 . Povoski SP. A prospective analysis of the cephalic vein cutdown approach for chronic indwelling central venous access in 100 consecutive cancer patients. Ann Surg Oncol 2000; 7:496-502.

7. Kincaid EH, Davis PW, Chang MC, et al. Blind placement of longterm central venous access devices: report of 589 consecutive procedures. Am Surg 1999; 65:520-554.

8. Wu CF, Po PJ, Wu CY, et al. A single-center study of vascular access sites for intravenous ports. Surg Today 2014; 44:723-731.

9. Aitken DR, Minton JP. The pinch-off sign": a warning of impending problems with permanent subclavian catheters. Am J Surg 1984; 148:633-63.

10. Hinke DH, Zandt-Stastny DA, Goodman LR, et al. Pinch-off syndrome: a complication of implantable subclavian venous access devices. Radiology 1990; 177:353-356.

11. Biswas S, Sidani M, Abrol S. Emergent median sternotomy for mediastinal hematoma: a rare complication following internal jugular vein catheterization for chemoport insertion-a case report and review of relevant literature. Case Rep Anesthesiol 2014; 190172.Epub.

12. Al-Mufarrej F, Martinez-Jorge J, Carlsen BT, et al. Use of the deltoid branch-based clavicular head of pectoralis major muscle flap in isolated sternoclavicular infections. J Plast Recontsr Aesthet Surg 2013; 66:1702-1711.

13. Okada $M$, Ikeda $M$, Uemura $T$, et al. A propeller flap based on the thoracoacrominal artery for reconstruction of a skin defect in the cervical region: a case report. J Plast Reconstr Aesthet Surg 2013; 66:720-722.

14. Onoda S, Sakuraba M, Asano T, et al. Thoracoacrominal vessels as recipients for head and neck reconstruction and case of vascular complications. Microsurgery 2011; 31:628-631.

15. Nagaso T, Shimizu Y, Kasai $S$, et al. Extension of jejunum in the reconstruction of cervical oesophagus with free jejunum transfer using the thoracoacrominal vessel as recipients. J Plast Recontsr Aesthet Surg 2012; 65:156-162.

16. Loukas M, Myers CS, Wartmann CT, et al. The clinical anatomy of the cephalic vein in the deltopectoral triangle. Folia Morphol (Warsz) 2008; 67:72-77.

17. Ta-Wei Su, Ching-Feng Wu, Jui-Ying Fu, Po-Jen Ko, Sheng-Yueh Yu, Tsung-Chi Kao, Hong-Chang Hsieh, and Ching-Yang Wu. Deltoid Branch of Thoracoacromial Vein: A Safe Alternative Entry Vessel for Intravenous Port Implantation. Medicine (Baltimore). 2015; 94(17): e728. 
Received: February 6, 2020

Accepted: June 18, 2020
Corresponding author

Sunita Kalra

net31aug@yahoo.co.in 